\title{
FUNCTION-THEORETIC CHARACTERIZATION OF EINSTEIN SPACES AND HARMONIC SPACES( $\left.{ }^{1}\right)$
}

\author{
BY \\ AVNER FRIEDMAN
}

Introduction. Consider the following general problem: Let $P$ be a function-theoretic property which holds at the points of a (local or global) Euclidean space $E_{n}$ and which can be stated, formally, for points of a Riemannian space $R_{n}$. Find a statement $Q$ which is an intrinsic (geometric) property of $R_{n}$ such that $P$ implies $Q$ and $Q$ implies $P$. Conversely, given $Q$, one may look for $P$.

In this paper we solve particular problems of the above type. We take for $Q$ the statements that $R_{n}$ (which is always assumed to have a positive definite metric) is an Einstein space, an harmonic space (to be defined in §3) with a particular fundamental solution, and a space with constant curvature. $P$ then stands for various statements about the mean value of solutions of certain equations. Denoting by $M\left(u, x^{0}, R\right)$ the mean value of $u$ on the geodesic sphere with center $x^{0}$ and radius $R$ we obtain the following results:

An Einstein space is characterized (in $\S 1$ ) by

$$
M\left(u, x^{0}, R\right)=u\left(x^{0}\right)\left(1+O\left(R^{3}\right)\right), \quad \frac{\partial}{\partial R} M\left(u, x^{0}, R\right)=u\left(x^{0}\right) O\left(R^{2}\right)
$$

for every $u \neq 0, \Delta u=0$ in a neighborhood of any of its points $x^{0}$, where $\Delta$ is the Laplace-Beltrami operator. Its scalar curvature $\rho$ is determined (in \$2) by (2.28) which holds for any $u \neq 0, \Delta u \neq 0, \Delta^{2} u=0$ in a neighborhood of any of its points $x^{0}$.

The characterization of an harmonic space by

$$
M\left(u, x^{0}, R\right)=u\left(x^{0}\right)
$$

for every solution $u$ of $\Delta u=0$ in a neighborhood of any of its points $x^{0}$ was already proved by Willmore [13]. The fundamental solution $\phi(r)$ of an harmonic space is determined (in $\S 3$ ) by a function $A(r)$ via the equation (3.4), whereas $A(R)$ is characterized by the mean value formula

$$
M\left(u, x^{0}, R\right)=u\left(x^{0}\right)+A(R) \Delta u\left(x^{0}\right)
$$

which holds for every solution $u$ of $\Delta^{2} u=0$ in a neighborhood of any point $x^{0}$.

A space of constant curvature $K$ is characterized (in $\$ 4$ ) by $(0.3)$ with $A(R)$ defined by

$$
A^{\prime}(R)=\int_{0}^{R}\left[\frac{\sin \left(R K^{1 / 2}\right)}{\sin \left(r K^{1 / 2}\right)}\right]^{n-1} d r, \quad A(0)=0 .
$$

Presented to the Society, January 24, 1961; received by the editors December 29, 1960 and, in revised form, February 17, 1961.

(1) Prepared under Contract Nonr 710(16) (NR 044 004) between the Office of Naval Research and the University of Minnesota. 
In $\$ 5$ we discuss the characterization of Einstein metrics by means of mean value theorems with regard to solutions of $\Delta u+\lambda u=0$ ( $\lambda$ constant) and also with regard to the set of eigenfunctions of $\Delta$. Analogous results are derived in $\$ 6$ for harmonic metrics.

General assumptions and notations. All the spaces are assumed to have a positive definite metric. The metric tensors are assumed to be sufficiently smooth. The dimension $n$ is taken to be $\geqq 3$; all the results however can easily be extended to the case $n=2$. We denote by $R_{n}$ a Riemannian space, by $A_{n}$ an Einstein space, by $H_{n}$ an harmonic space and by $K_{n}$ a space of constant curvature. All the considerations of this paper are local.

1. Characterization of Einstein spaces. Let $\left(g_{i j}\right)$ be the metric tensor of $R_{n}$. The Laplace-Beltrami operator is defined by

$$
\Delta u=\frac{1}{g^{1 / 2}} \frac{\partial}{\partial x^{i}}\left(g^{1 / 2} g^{i j} \frac{\partial u}{\partial x^{i}}\right)=g^{i j} u, i j \quad\left(g=\operatorname{det}\left(g_{i j}\right)\right)
$$

where "comma" denotes covariant differentiation. Let $x^{0}$ be a fixed point in $R_{n}$ and let $r$ denote the geodesic distance from $x^{0}$ to a variable point $x$ in $R_{n}$. Since our considerations are local, we shall assume, for simplicity, throughout this paper, that $r$ exists for all $x^{0}, x$ in $R_{n}$. We say that $R_{n}$ is Einsteinian at $x^{0}$ if

$$
R_{i j}=k g_{i j} \quad \text { at } \quad x=x^{0},
$$

where $R_{i j}$ is the Ricci tensor and $k$ is a constant.

LEMMA 1. $R_{n}$ is Einsteinian at $x^{0}$ if and only if

$$
\Delta r=\frac{n-1}{r}+\beta r+F
$$

where $\beta$ is a constant and $F=O\left(r^{2}\right)$ as $r \rightarrow 0$.

REMARK. The lemma and the proof are valid also when the metric of $R_{n}$ is indefinite.

Proof $\left({ }^{2}\right)$. Let $\Theta^{i}$ be the unit vector at $x^{0}$ tangent to the geodesic which connects $x^{0}$ to $x$. Then

$$
x^{i}=\Theta^{i r}
$$

are normal coordinates of $x$ about $x^{0}$ as the origin. Let $\left(\bar{g}_{i j}\right)$ be the metric tensor in the $x^{i}$ coordinates. Denoting by $\left(g_{i j}\right)_{0}$ the value of $\left(g_{i j}\right)$ at $x^{0}\left(\left(g_{i j}\right)_{0}=\left(\bar{g}_{i j}\right)_{0}\right)$ we have $[11$, p. 96$]$

$$
r^{2}=\left(g_{i j}\right)_{0} x^{i} x^{j}=\bar{g}_{i j} x^{i} x^{j}
$$

and

(2) The author thanks the referee for suggesting the present proof which is somewhat simpler than the author's original proof. 


$$
r r, i=\left(g_{i j}\right)_{0} x^{i}=\bar{g}_{i j} x^{j} \quad\left(r, i=\frac{\partial r}{\partial x^{i}}\right) .
$$

Hence,

$$
r^{i}=x^{i} \quad\left(\boldsymbol{r}^{i}=\overline{\mathrm{g}}^{i{ }^{i} \boldsymbol{r}, j}\right) .
$$

Differentiating (1.5) covariantly we get

$$
\left(r r^{i}\right), j=\delta_{j}^{i}+\Gamma_{k j}^{i} x^{k}
$$

where $\Gamma_{k t}^{i}$ are Christoffel's symbols with respect to $\left(\bar{g}_{i j}\right)$.

Contracting $i, j$ :

$$
r \Delta r+r, r^{i}=n+\Gamma_{k i}^{i} x^{k} .
$$

Using $r, i r^{i}=1$ (which follows from (1.4), (1.3)), we get

$$
r \Delta r=n-1+\Gamma_{k i}^{i} x^{k}
$$

But

$$
\Gamma_{k i}^{i}=\left(\Gamma_{k i}^{i}\right)_{0}+\left(\frac{\partial \Gamma_{k i}^{i}}{\partial x^{j}}\right)_{0}^{j}+O\left(r^{2}\right)
$$

and

$$
\frac{\partial \Gamma_{k i}^{i}}{\partial x^{j}}=\frac{1}{3} R_{k j} \text { at } x^{0}
$$

(which follows using the identity $\partial \Gamma_{h m}^{i} / \partial x^{k}+\partial \Gamma_{m k}^{i} / x^{h}+\partial \Gamma_{k n}^{i} / \partial x^{m}=0$ at $x^{0}$; see $[4$, p. 52]). Hence we obtain from (1.7)

$$
\Delta r=\frac{n-1}{r}+\frac{1}{3}\left(R_{i j}\right)_{0} \Theta^{i} \Theta^{i} r+F, \quad F=O\left(r^{2}\right) .
$$

Using (1.8), the proof of the lemma follows easily.

Indeed, if $R_{n}$ is Einsteinian at $x^{0}$ then

$$
\left(R_{i j}\right)_{0}=k\left(g_{i j}\right)_{0}
$$$$
\text { ( } k=\text { const }) \text {. }
$$

Since, by (1.2), (1.3),

$$
\left(g_{i j}\right)_{0} \Theta^{i} \Theta^{i}=1,
$$

(1.1) follows with $\beta=k / 3$. 
Conversely, if (1.1) holds, then by (1.8)

$$
\frac{1}{3}\left(R_{i j}\right)_{0} \Theta^{i} \Theta^{j}=\beta
$$

for all $\Theta^{k}$ satisfying (1.10). Hence (1.9) follows with $k=3 \beta$. This completes the proof of the lemma.

Let $u^{a}(a=1, \cdots, n-1)$ be local coordinates on the unit sphere. Then $r, u^{a}$ form polar geodesic coordinates about $x^{0}$, and we have

$$
\begin{aligned}
& d s^{2}=d r^{2}+\gamma_{a b}\left(r, u^{c}\right) d u^{a} d u^{b} \\
& \Delta U=\frac{1}{\gamma^{1 / 2}} \frac{\partial}{\partial r}\left(\gamma^{1 / 2} \frac{\partial U}{\partial r}\right)+\frac{1}{\gamma^{1 / 2}} \frac{\partial}{\partial u^{a}}\left(\gamma^{1 / 2} \gamma^{a b} \frac{\partial U}{\partial u^{b}}\right),
\end{aligned}
$$

where $\gamma=\operatorname{det}\left(\gamma_{a b}\right)$. Hence,

$$
\Delta r=\frac{1}{\gamma^{1 / 2}} \frac{\partial \gamma^{1 / 2}}{\partial r}=\frac{\partial}{\partial r}\left(\log \gamma^{1 / 2}\right) .
$$

The last two formulas will be needed later on.

Let $S_{R}$ be the geodesic sphere $r=R$ and denote its interior by $D_{R}$. The mean value of a function $u$ is defined by

$$
M\left(u, x^{0}, R\right)=\int_{S_{R}} u d \sigma / \int_{S_{R}} d \sigma
$$

where $d \sigma=\gamma^{1 / 2} d u^{1} \cdots d u^{n-1}$ is the surface element of area. For brevity we also set

$$
M(R)=M\left(u, x^{0}, R\right), \quad S(R)=\int_{S_{R}} d \sigma .
$$

Theorem 1. A necessary and sufficient condition that $R_{n}$ be Einsteinian at a point $x^{0}$ is that for every solution $u$ of $\Delta u=0$ in some neighborhood $D_{R_{u}}$ of $x^{0}$ such that $u \neq 0$ in $D_{R_{u}}$,

$$
M\left(u, x^{0}, R\right)=u\left(x^{0}\right)\left(1+O\left(R^{3}\right)\right), \quad \frac{\partial}{\partial R} M\left(u, x^{0}, R\right)=u\left(x^{0}\right) O\left(R^{2}\right)
$$

for $R \leqq R_{u}$, where $O\left(R^{k}\right)(k=2,3)$ is defined for $R \leqq R_{u}$ and $\left|O\left(R^{k}\right)\right| / R^{k} \leqq A$ where $A$ is a constant independent of $u, R, R_{u}$.

Proof. Differentiating $M(R) \int_{S_{R}} d \sigma=\int_{S_{R}} u d \sigma$ and using (1.13) and $\int_{D_{R}} \Delta u d V$ $=\int_{S_{R}}(\partial u / \partial \nu) d \sigma$, where $\nu$ is the outwardly directed normal to $S_{R}$, we obtain

$$
S(R) M^{\prime}(R)+M(R) \int_{S_{R}} \Delta R d \sigma=\int_{S_{R}} u \Delta R d \sigma+\int_{D_{R}} \Delta u d V
$$


This identity holds for each point $x^{0} \in R_{n}$ and for any function $u$ (it was used by Willmore [13]).

Suppose now that $R_{n}$ is Einsteinian at $x^{0}$. Then, we can substitute $\Delta r$ from (1.1) into (1.17). Using also the fact that $\Delta u=0,(1.17)$ becomes

$$
S(R) M^{\prime}(R)+M(R) \int_{S_{R}} F d \sigma=\int_{S_{R}} u F d \sigma .
$$

Let $u \neq 0$ in a neighborhood $D_{R_{x}}$ of $x^{0}$. It is enough to consider the case $u>0$, since in the case $u<0$ we first obtain the mean value formula for $-u$ and then change the sign of both sides. Using $u>0$ and $F=O\left(R^{2}\right)$ on $S_{R}$, we derive from (1.18),

$$
\frac{M^{\prime}(R)}{M(R)}=O\left(R^{2}\right), \quad \text { or } \quad \log \frac{M(R)}{M(0)}=O\left(R^{3}\right) .
$$

Since $M(0)=u\left(x^{0}\right)$ and $\exp \left\{O\left(R^{3}\right)\right\}=1+O\left(R^{3}\right)$, we get

$$
M(R)=u\left(x^{0}\right)\left(1+O\left(R^{3}\right)\right) .
$$

Substituting this into (1.18) we find (using: $u>0$ ) that $M^{\prime}(R)=u\left(x^{0}\right) O\left(R^{2}\right)$, and the proof of (1.16) is completed.

Suppose conversely that (1.16) is satisfied. Then, (1.17) yields, if $\Delta u=0$,

$$
u\left(x^{0}\right) S(R) O\left(R^{2}\right)+\frac{\delta(R)}{S(R)} \int_{S_{R}} u d \sigma=\int_{S_{R}} u \Delta R d \sigma
$$

where

$$
\delta(R)=\int_{S_{R}} \Delta R d \sigma .
$$

Expressing $u\left(x^{0}\right)$ (by (1.16)) in terms of $M(R)$, we get

$$
\int_{S_{R}} u\left[\Delta R-\frac{\delta(R)}{S(R)}+H\left(R, u^{a}\right)\right] d \sigma=0
$$

and $\left|H\left(R, u^{a}\right)\right| / R^{2} \leqq A_{0}$, where $A_{0}$ is a constant independent of $u, R$.

By Lemma 1, all we have to prove is that (1.1) holds (where $\beta$ is a constant). If (1.1) does not hold then, using (1.8) and $\delta(r) / S(r)=(n-1) / r+k_{0} r$ $+O\left(r^{2}\right), k_{0}=$ const. (which follows (1.8), (1.20)), we conclude that there exists a cone $K$ with positive opening and vertex $x^{0}$ such that

$$
\Delta r-\frac{\delta(r)}{S(r)}>\epsilon_{0} r \quad\left(\text { or } \leqq-\epsilon_{0} r\right) \text { in } K,
$$

where $\epsilon_{0}>0$. It will be enough to consider the case $>\epsilon_{0} r$. 
Set $K_{R}=K \cap S_{R}, K(R)=$ area of $K_{R}$ and let $g_{R}$ be a non-negative smooth function defined on $S_{R}$ such that $g_{R}=1$ on $K_{R}$ and $g_{R}=0$ outside a sufficiently small neighborhood of $K_{R}$ (on $S_{R}$ ) in which the first inequality of (1.22) still holds. Let $u_{R}$ be the solution of $\Delta u_{R}=0$ in $D_{R}$ which assumes the values of $g_{R}$ on $S_{R}$. Then $u_{R}$ is positive in $D_{R}$, and an application of (1.21) gives

$$
K(R) \epsilon_{0} R-S(R) A_{0} R^{2}<0
$$

which is a contradiction if $R$ is sufficiently small.

Using $\Delta r=(n-1) / r+O(r)$ in the proof of the first part of Theorem 1, we get:

TheOREM 2. At any point $x^{0}$ of $R_{n}$,

$$
M\left(u, x^{0}, R\right)=u\left(x^{0}\right)\left(1+O\left(R^{2}\right)\right), \quad \frac{\partial}{\partial R} M\left(u, x^{0}, R\right)=u\left(x^{0}\right) O(R)
$$

for any solution $u$ of $\Delta u=0$ in $D_{R_{u}}\left(u \neq 0\right.$ in $\left.D_{R_{u}}\right) . O\left(R^{k}\right)$ is defined for $R \leqq K_{u}$ and $\left|O\left(R^{k}\right)\right| / R^{k} \leqq A(k=1,2)$ where $A$ is independent of $u, R, R_{u}$.

REMARK 1. From the proof of the first part of Theorem 1 it follows that for every $R_{n}$, if $u \geqq 0, \Delta u=0$, then

$$
M^{\prime}(R)+M(R) O(R)=0 .
$$

Suppose now that $u\left(x^{0}\right)=0$. Then $M(0)=0$ and we can write (1.25) as a Volterra type integral equation

$$
M^{\prime}(R)+O(R) \int_{0}^{R} M^{\prime}(t) d t=0 .
$$

We conclude that $M^{\prime}(R) \equiv 0$ and, hence, $M(R) \equiv 0$. Since $u \geqq 0$, it follows that $u \equiv 0$. We have thus proved: In every $R_{n}$, if $u\left(x^{0}\right)=0, u \geqq 0, \Delta u=0$, then $u \equiv 0$. This is the well known maximum principle for solutions of the LaplaceBeltrami equation.

REMARK 2. From the proof of Theorem 1 it follows (see (1.23)) that for the "sufficiency" part of the theorem it is enough to assume that (1.16) holds with $O\left(R^{2}\right)$ and $O\left(R^{3}\right)$ replaced by $o(R)$ and $o\left(R^{2}\right)$, respectively.

REMARK 3 . The conditions (1.16) are consequences of the single condition

$$
L M(R) \equiv M^{\prime \prime}(R)+\frac{n-1}{R} M^{\prime}(R)=M(R) O(R) .
$$

Indeed, integrating (1.26) we get $M^{\prime}(R)=\left(\int_{0}^{R} M(t) d t\right) O(R)$. Hence, $M(R)$ $=u\left(x^{0}\right)+\left(\int_{0}^{R} M(t) d t\right) O\left(R^{2}\right)$. This leads to $M(R)=u\left(x^{0}\right)\left(1+O\left(R^{3}\right)\right)$. Finally, $M^{\prime}(R)=\left(\int_{0}^{R} M(t) d t\right) O(R)=u\left(x^{0}\right) O\left(R^{2}\right)$.

Note that $L$ is the radial part of the Laplace operator in $n$ variables. 
2. Determination of the scalar curvature. In this section we assume that $R_{n}$ is Einsteinian at a point $x^{0}$ and determine the scalar curvature at this point. We shall need a well known integral formula (see $[3$, p. 112]) which holds in any $R_{n}$ : Let

$$
\gamma\left(x, x^{0}\right)=\gamma_{n} r^{2-n}+O\left(r^{3-n}\right), \quad \frac{\partial}{\partial x^{i}} \gamma\left(x, x^{0}\right)=(2-n) \gamma_{n} r^{-n} x^{i}+O\left(r^{2-n}\right)
$$

as $r \rightarrow 0$, where $O\left(r^{0}\right)$ is always understood to mean $O(\log (1 / r))$, and where

$$
\gamma_{n}=\frac{1}{(n-2) \omega_{n}}, \quad \omega_{n}=\frac{2 \pi^{n / 2}}{\Gamma(n / 2)}
$$

and let $\Delta \gamma\left(x, x^{0}\right)$ be integrable. $\gamma$ is assumed to be smooth for $x \neq x^{0}, x^{0}$ being a fixed point in a domain $D$ with smooth boundary $B$. Then, for any smooth function $u$ in $D+B$,

$$
\begin{aligned}
u\left(x^{0}\right)= & \int_{D}\left[u(x) \Delta \gamma\left(x, x^{0}\right)-\gamma\left(x, x^{0}\right) \Delta u(x)\right] d V_{x} \\
& +\int_{B}\left[\gamma\left(y, x^{0}\right) \frac{\partial u(y)}{\partial \nu}-u(y) \frac{\partial \gamma\left(y, x^{0}\right)}{\partial \nu}\right] d \sigma_{\gamma}
\end{aligned}
$$

where $\partial / \partial \nu$ is the derivative in the direction of the outward normal to $B$ at $y$.

Now let $\phi(r)$ be a solution of the equation

$$
\phi^{\prime \prime}+\left(\frac{n-1}{r}+\beta r\right) \phi^{\prime}=0
$$

where $\beta$ appears in $\S 1 . \phi$ is determined up to an additive constant if we require that $\phi(r) r^{n-2} \rightarrow$ prescribed number, as $r \rightarrow 0$. For the sake of definiteness we always take this number to be 1 . Then

$$
\phi^{\prime}(r)=(2-n) r^{1-n} \exp \left\{-\frac{1}{2} \beta r^{2}\right\}
$$

and

$$
\phi(r)=r^{2-n}+O\left(r^{4-n}\right), \quad \phi^{\prime}(r)=(2-n) r^{1-n}+O\left(r^{3-n}\right) .
$$

By (1.12), (1.13), $\Delta \psi(r)=\psi^{\prime \prime}(r)+\psi^{\prime}(r) \Delta r$ for any function $\psi(r)$. Using (2.2), (1.1) we find that

$$
\Delta \phi=\phi^{\prime \prime}+\phi^{\prime} \Delta r=\phi^{\prime} F=O\left(r^{3-n}\right) .
$$

Applying (2.1) with

$$
\gamma\left(x, x^{0}\right)=\Phi(r)-\Phi(R) \text { where } \Phi(r)=\gamma_{n} \phi(r)
$$

and with $D$ being $D_{R}$, we obtain 
(2.7) $u\left(x^{0}\right)=\int_{D_{R}} \Phi^{\prime} F u d V-\int_{D_{R}}[\Phi(r)-\Phi(R)] \Delta u d V-\int_{S_{R}}\left[u \frac{d \Phi}{d r}\right]_{r=R} d \sigma$, since $\partial \Phi / \partial \nu=d \Phi / d r$ on $r=R$. Taking $u \equiv 1$ in (2.7) we get

$$
1+O\left(R^{3}\right)=-\left[\frac{d \Phi}{d r}\right]_{r=R} \int_{S_{R}} d \sigma .
$$

Substituting $d \Phi / d r$ from (2.8) into (2.7) we obtain

$$
\begin{aligned}
& M\left(u, x^{0}, R\right)\left(1+O\left(R^{3}\right)\right) \\
& \quad=u\left(x^{0}\right)-\int_{D_{R}} \Phi^{\prime} F u d V+\int_{D_{R}}[\Phi(r)-\Phi(R)] \Delta u d V .
\end{aligned}
$$

In order to evaluate the third term on the right side of (2.9), we introduce a function $v$ which satisfies:

$$
\begin{gathered}
v^{\prime \prime}+\left(\frac{n-1}{r}+\beta r\right) v^{\prime}=\Phi(r)-\Phi(R), \\
v(R)=0, \quad v^{\prime}(R)=0 .
\end{gathered}
$$

We can write (2.10) in the form (see (2.2))

$$
v^{\prime \prime}(r)-\frac{\phi^{\prime \prime}(r)}{\phi^{\prime}(r)} v^{\prime}(r)=\gamma_{n}[\phi(r)-\phi(R)],
$$

or

$$
\left(\frac{v^{\prime}(r)}{\phi^{\prime}(r)}\right)^{\prime}=\gamma_{n} \frac{\phi(r)}{\phi^{\prime}(r)}-\gamma_{n} \frac{\phi(R)}{\phi^{\prime}(r)} .
$$

Integrating and making use of (2.11) we get

$$
\begin{aligned}
-v^{\prime}(r)= & \gamma_{n} \phi^{\prime}(r) \int_{r}^{R} \frac{\phi(\lambda)}{\phi^{\prime}(\lambda)} d \lambda-\gamma_{n} \phi(R) \phi^{\prime}(r) \int_{r}^{R} \frac{d \lambda}{\phi^{\prime}(\lambda)} \\
v(t)= & \gamma_{n} \int_{t}^{R} \phi^{\prime}(r)\left(\int_{r}^{R} \frac{\phi(\lambda)}{\phi^{\prime}(\lambda)} d \lambda\right) d r \\
& -\gamma_{n} \int_{t}^{R} \phi(R) \phi^{\prime}(r)\left(\int_{r}^{R} \frac{d \lambda}{\phi^{\prime}(\lambda)}\right) d r .
\end{aligned}
$$

Using only the fact that $\phi$ satisfies (2.4), one easily derives from (2.14) and (2.15):

$$
v(r)=\gamma_{n} A(R) r^{2-n}+O\left(r^{3-n}\right), \quad v^{\prime}(r)=(2-n) \gamma_{n} A(R) r^{1-n}+O\left(r^{2-n}\right)
$$

where 


$$
A(R)=-\int_{0}^{R} \frac{\phi(\lambda)}{\phi^{\prime}(\lambda)} d \lambda+\phi(R) \int_{0}^{R} \frac{d \lambda}{\phi^{\prime}(\lambda)}
$$

or, equivalently,

$$
A^{\prime}(R)=\phi^{\prime}(R) \int_{0}^{R} \frac{d \lambda}{\phi^{\prime}(\lambda)}, \quad A(0)=0
$$

Later we shall use the estimate

$$
v^{\prime}(r)=A(R) O\left(r^{1-n}\right)
$$

which follows from (2.16), noting that (by (2.18), (2.4)) $A(R)=$ const. $R^{2}$ $+O\left(R^{3}\right)$.

Substituting $\phi$ from (2.3) into (2.18), we obtain

$$
A(R)=\frac{R^{2}}{2 n}-\frac{\beta B^{4}}{4 n(n+2)}+O\left(R^{6}\right)
$$

We now use (2.1) with $u$ replaced by $\Delta u, \gamma$ being $v$ and $D=D_{R}$. Using (2.11), (2.16) and

$$
\Delta v(r)=v^{\prime \prime}(r)+v^{\prime}(r) \Delta r=[\Phi(r)-\Phi(R)]+v^{\prime}(r) F,
$$

we obtain

$$
A(R) \Delta u\left(x^{0}\right)=\int_{D_{R}}[\Phi(r)-\Phi(R)] \Delta u d V+\int_{D_{R}}{ }^{\prime} F \Delta u d V-\int_{D_{R}}{ }^{\prime} \Delta^{2} u d V
$$

If $\Delta^{2} u=0$ then, by comparing (2.21) with (2.9), we get

$$
\begin{aligned}
M\left(u, x^{0}, R\right)(1 & \left.+O\left(R^{8}\right)\right) \\
& =u\left(x^{0}\right)-\int_{D_{R}} \phi^{\prime} F u d V+A(R) \Delta u\left(x^{0}\right)+\int_{D_{R}} v^{\prime} F \Delta u d V .
\end{aligned}
$$

We now assume that $u \neq 0, \Delta u \neq 0$ in some $D_{R_{u}}$ and take $R \leqq R_{u}$. We then have:

$$
\begin{aligned}
-\int_{D_{R}} \phi^{\prime} F u d V & =\int_{0}^{R} O\left(r^{8-n}\right)\left(\int_{S_{r}} u d \sigma\right) d r=\int_{0}^{R} O\left(r^{8-n}\right) S(r) M(r) d r \\
& =O\left(R^{2}\right) \int_{0}^{R} M(r) d r
\end{aligned}
$$

$$
\begin{aligned}
\int_{D_{R}} v^{\prime} F \Delta u d V & =\int_{D_{R}} A(r) O\left(r^{1-n}\right) O\left(r^{2}\right) \Delta u d V \\
& =A(R) \int_{0}^{R} O\left(r^{r-n}\right)\left(\int_{S_{r}} \Delta u d \sigma\right) d r
\end{aligned}
$$


(using $\left(2.16^{\prime}\right)$ ), and since $\Delta(\Delta u)=0, \Delta u \neq 0$, we can apply Theorem 1 and get

$$
\int_{D_{R}} v^{\prime} F \Delta u d V=A(R) O\left(R^{3}\right) \Delta u\left(x^{0}\right) .
$$

Substituting (2.23), (2.24) into (2.22), (2.22) simplifies to

$$
\begin{aligned}
& M(R)+O\left(R^{2}\right) \int_{0}^{R} M(r) d r \\
& \quad=u\left(x^{0}\right)\left(1+O\left(R^{3}\right)\right)+A(R) \Delta u\left(x^{0}\right)\left(1+O\left(R^{3}\right)\right) .
\end{aligned}
$$

Integrating (2.25) over $R$ we find that

$$
\int_{0}^{R} M(r) d r=u\left(x^{0}\right) O(R)+A(R) \Delta u\left(x^{0}\right) O(R) .
$$

Hence, (2.25) reduces to

$$
M(R)=u\left(x^{0}\right)\left(1+O\left(R^{3}\right)\right)+A(R) \Delta u\left(x^{0}\right)\left(1+O\left(R^{3}\right)\right),
$$

and substituting $A(R)$ from (2.19), we get

$$
M\left(u, x^{0}, R\right)=u\left(x^{0}\right)\left(1+O\left(R^{3}\right)\right)+u\left(x^{0}\right)\left\{\frac{R^{2}}{2 n}-\frac{\beta R^{4}}{4 n(n+2)}+O\left(R^{5}\right)\right\} .
$$

Note that the proof of (2.27) remains true also in case $u\left(x^{0}\right)=0$, provided either $u \geqq 0$ or $u \leqq 0$.

From (1.9) and $k=3 \beta$ it follows that $\rho=3 \beta n$ is the scalar curvature at $x^{0}$. We have thus proved:

THEOREM 3. Let $R_{n}$ be Einsteinian at a point $x^{0}$. Then for any function $u$ defined in some neighborhood $R_{R_{u}}$ of $x^{0}$ and satisfying $u \geqq 0$ or $u \leqq 0, \Delta u \neq 0$, $\Delta^{2} u=0$ in $D_{R_{v}}$, we have:

$$
M\left(u, x^{0}, R\right)=u\left(x^{0}\right)\left(1+O\left(R^{3}\right)\right)+\Delta u\left(x^{0}\right)\left(\frac{R^{2}}{2 n}+\frac{\rho R^{4}}{12 n^{2}(n+2)}+O\left(R^{5}\right)\right)
$$

where $O\left(R^{k}\right)(k=3,5)$ is defined for $R \leqq R_{u}$ and satisfies $\left|O\left(R^{k}\right)\right| / R^{k} \leqq B$, where $B$ is a constant independent of $u, R, R_{u}$. The coefficient $\rho$ is the scalar curvature of $R_{n}$ at $x^{0}$.

REMARK 1. In order to calculate $\rho$ from (2.28) one should use it with a function $u$ which vanishes at $x^{0}$.

REMARk 2. Set

$$
\begin{aligned}
f(\Theta) & =\frac{1}{3}\left(R_{i j}\right)_{0} \Theta^{i} \Theta^{j}, & f_{0} & =\lim _{R \rightarrow 0} M\left(f, x^{0}, R\right), \\
f_{1} & =\text { g.l.b. } f(\Theta), & f_{2} & =\text { l.u.b. } f(\Theta) .
\end{aligned}
$$


Then, using (2.1) with $\gamma=r^{2-n}-R^{2-n}$ we easily obtain, if $\Delta u=0$ and $u>0$,

$$
1+\frac{R^{2}}{2 \gamma_{n}}\left(f_{1}-f_{0}\right)+O\left(R^{3}\right) \leqq \frac{M\left(u, x^{0}, R\right)}{u\left(x^{0}\right)} \leqq 1+\frac{R^{2}}{2 \gamma_{n}}\left(f_{2}-f_{0}\right)+O\left(R^{3}\right)
$$

which sharpens Theorem 2. Note that $\rho=3 n f_{0}$.

3. Characterization of harmonic spaces. A Riemannian space $R_{n}$ is said to be harmonic at a point $x^{0}$ if the equation $\Delta u=0$ has a solution $u=\phi(r)$ in some neighborhood of $x^{0}$. The space is trivially harmonic (or simply harmonic) at $x^{0}$ if $\phi(r)=r^{2-n}$. If $R_{n}$ is (trivially)harmonic at each of its points, then we say that $R_{n}$ is (trivially)harmonic. It is easily seen that $\phi(r)$ is a fundamental solution of $\Delta u=0$. The theory of harmonic spaces was developed by Ruse, Walker, Lichnerowicz, and others. For a general survey see [7] and for related references, see [14]. We mention that $H_{n}$ is necessarily an $A_{n}$, whereas every space with constant curvature $K_{n}$ is necessarily an $H_{n}$. For $n=2,3$, $A_{n}=H_{n}=K_{n}$. The last statement is also true in the case of indefinite metric tensors.

Willmore [13] proved that a necessary and sufficient condition for $R_{n}$ to be harmonic at a point $x^{0}$ is that for every solution $u$ of $\Delta u=0$, in some neighborhood of $x^{0}$,

$$
M\left(u, x^{0}, R\right)=u\left(x^{0}\right)
$$

We now want to find a characterization for the fundamental solution $\phi(r)$.

THEOREM 4. If $R_{n}$ is harmonic at a point $x^{0}$, then for any solution $u$ of $\Delta^{2} u$ $=0$, in some neighborhood $D_{R_{u}}$ of $x^{0}$,

$$
M\left(u, x^{0}, R\right)=u\left(x^{0}\right)+A(R) \Delta u\left(x^{0}\right)
$$

where $A(R)$ is related to $\phi(R)$ by

$$
A(R)=-\int_{0}^{R} \frac{\phi(\lambda)}{\phi^{\prime}(\lambda)} d \lambda+\phi(R) \int_{0}^{R} \frac{d \lambda}{\phi^{\prime}(\lambda)}
$$

or, equivalently, by

$$
A^{\prime}(R)=\phi^{\prime}(R) \int_{0}^{R} \frac{d \lambda}{\phi^{\prime}(\lambda)}, \quad A(0)=0 .
$$

If $R_{n}$ is an $H_{n}$ then [7] $\phi(r)$ is independent of the initial point $x^{0}$. Therefore, the same is true of $A(R)$.

REMARK. Since we assume that $\phi(r)$ is normalized by $\phi(r)=r^{2-n}+O\left(r^{3-n}\right)$, it is determined by (3.4) up to an additive constant. Indeed, this follows from the equation 


$$
\frac{\phi^{\prime \prime}}{\phi^{\prime}}=\frac{A^{\prime \prime}-1}{A^{\prime}}
$$

which is derived from (3.4).

Proof of Theorem 4. The proof is similar to the proof of Theorem 3 and we therefore only indicate the modifications which one has to make. (2.5) is replaced by $\Delta \phi=0$ and hence in (2.7), (2.9) $F \equiv 0$. Also $O\left(R^{3}\right) \equiv 0$ in (2.8), (2.9). As for $v$, the only difference is that instead of $(n-1) / r+\beta r$ (in (2.10)) we now write $\Delta r$ or $-\phi^{\prime \prime} / \phi^{\prime}$. Consequently, in (2.20) we have $F \equiv 0$.

REMARK 1. Helgason [6] has recently extended Ásgeirson's mean value theorem [1] to solutions of $\Delta_{x} u=\Delta_{y} u$, where $\Delta_{x}, \Delta_{y}$ are the Laplace-Beltrami operators in the $x$-space and the $y$-space of $\boldsymbol{n}$-dimensions. He assumed that the spaces are two-point homogeneous spaces (and, hence, harmonic). It is immediately seen (using [13]) that for Ásgeirson's theorem to be valid it is necessary that the two spaces be harmonic.

REMARK 2. In 1909 Pizetti proved (see [2, p. 261]) that for any smooth function $u$ and a non-negative integer $m$,

$$
\frac{1}{S(R)} \int_{S_{R}} u d \sigma=\Gamma\left(\frac{n}{2}\right) \sum_{k=0}^{m}\left(\frac{R}{2}\right)^{2 k} \frac{\Delta^{k} u\left(x^{0}\right)}{k ! \Gamma(k+n / 2)}-\int_{D_{R}} v_{m} \Delta^{m+1} u d V
$$

where

$$
\begin{aligned}
v_{0} & =\gamma_{n}\left(r^{2-n}-R^{2-n}\right), \\
v_{k}(r) & =\gamma_{n} \int_{r}^{R} \rho v_{k-1}(\rho)\left[\rho^{n-2}-r^{n-2}\right] d \rho,
\end{aligned}
$$

if $n \neq 2$. For $n=2$ the definition of $v_{k}$ is slightly different. The proof can easily be extended to $R_{n}$ harmonic at $x^{0}$, provided

$$
\begin{aligned}
& 0<A_{1} \leqq r^{n-1} \phi^{\prime}(r) \leqq A_{2} \\
& 0<A_{3} \leqq \frac{r^{n-2} R}{R-r}[\phi(r)-\phi(R)] \leqq A_{4}
\end{aligned}
$$

The formula obtained is

$$
M\left(u, x^{0}, R\right)=\sum_{k=0}^{m} A_{k}(R) \Delta^{k} u\left(x^{0}\right)-\int_{D_{R}} v_{m} \Delta^{m+1} u d V
$$

and

$$
0<B_{0} B_{1}^{k} \leqq \frac{A_{k}(R)}{R^{2 k}(k !)^{2}} \leqq B_{2} B_{3}^{k}
$$




$$
0<v_{m}(r) \leqq B_{4} B_{5}^{m} \frac{(R-r)^{2 m+1}}{R(m !)^{2}} r^{2-n}
$$

where the $B_{i}$ are constants depending only on the $A_{i}$.

Formula (3.8) with the estimates (3.9) can be used to derive a Liouville type theorem, namely: if $\Delta^{p} u=0$ ( $p$ a positive integer) in the whole Euclidean space, where $\Delta$ is the Laplace-Beltrami operator, and if $u$ is bounded, then $u \equiv$ const. (We assume that (3.6), (3.7) hold for all $0<r<R<\infty$ and that $\Delta$ is uniformly elliptic.) The proof is obtained by first deducing that $\Delta^{p-1} u=0$, $\Delta^{p-2} u=0, \cdots, \Delta u=0$ and then applying Nash's estimates [9] which immediately give $u \equiv$ const.

4. Characterization of spaces of constant curvature. We need the following lemma:

LEMMA 2. If in a space $H_{n}$

$$
\phi^{\prime}(r)=(2-n)\left[\frac{K^{1 / 2}}{\sin \left(r K^{1 / 2}\right)}\right]^{n-1}, \quad K \neq 0(K \text { constant })
$$

then $H_{n}$ is a space of constant curvature $K$.

The converse of this lemma is well known [7] and is proved by direct calculation. Lemma 2 for $K=0\left((4.1)\right.$ is then understood to mean: $\phi^{\prime}(r)$ $\left.=(2-n) r^{1-n}\right)$ is due to Thomas and Titt [10].

Proof. Set

$$
\chi(r)=-\frac{\phi^{\prime \prime}(r)}{\phi^{\prime}(r)}, \quad f(\Omega)=r \chi(r)+1 \text { where } \Omega=\frac{1}{2} r^{2} .
$$

Lichnerowicz [7] (and later also Willmore [12]) proved that for any $H_{n}$

$$
\frac{5}{2} f^{\prime \prime}(0)+\frac{\left(f^{\prime}(0)\right)^{2}}{n-1} \leqq 0
$$

and equality holds if and only if $H_{n}$ is a $K_{n}$; its curvature $K$ is then found to be

$$
K=-\frac{3 f^{\prime}(0)}{2(n-1)} \text {. }
$$

A simple calculation shows that for $\phi^{\prime}$ as given by (4.1) we have

$$
f^{\prime}(0)=-\frac{2(n-1)}{3} K, \quad f^{\prime \prime}(0)=-\frac{8(n-1)}{45} K^{2} .
$$

Hence equality holds in (4.2) and the proof of Lemma 2 is completed.

Combining Theorem 4 and Lemma 2, we get: 
THEOREM 5. A necessary and sufficient condition for an $R_{n}$ to be $a K_{n}$ with curvature $K$ is that (3.2) holds at each point $x^{0}$ of $R_{n}$ and for every solution $u$ of $\Delta^{2} u=0$ in a neighborhood of $x^{0}$, where $A(R)$ is given by

$$
A^{\prime}(R)=\int_{0}^{R}\left[\frac{\sin \left(r K^{1 / 2}\right)}{\sin \left(R K^{1 / 2}\right)}\right]^{n-1} d r, \quad A(0)=0 .
$$

REMARK. Using (3.5) we have

$$
f(\Omega)=r \frac{1-A^{\prime \prime}(r)}{A^{\prime}(r)}+1, \quad \Omega=\frac{1}{2} r^{2} .
$$

We can use this formula to calculate $f^{\prime}(0), f^{\prime \prime}(0)$ in terms of $A^{(4)}(0), A^{(6)}(0)$ (note that $A^{(2 m+1)}(0)=0$ for $\left.m=0,1,2, \cdots\right)$. We then can express (4.2) as an inequality involving $A^{(4)}(0), A^{(6)}(0)$. In particular, if $A^{(4)}(0)=A^{(6)}(0)$ $=0$ then $H_{n}$ is flat.

5. Characterization of Einstein metrics, using solutions of $\Delta u+\lambda u=0$. The following theorem can be derived by the method of $\S 1$ :

$R_{n}$ is Einsteinian at $x^{0}$ if and only if for every solution $u \geqq 0$ of $\Delta u+\lambda u$ $=0(\lambda$ fixed $)$ in some $D_{R_{u}}$,

$$
M^{\prime}(R)+\frac{\lambda}{S(R)} \int_{0}^{R} S(r) M(r) d r=O\left(R^{2}\right) M(R)
$$

for $R \leqq R_{u}$, where $\left|O\left(R^{2}\right)\right| \leqq A R^{2}, A$ being independent of $u, R_{u}, R$.

Let $\left\{\lambda_{m}\right\},\left\{\phi_{m}\right\}$ be the sets of eigenvalues and orthonormal eigenfunctions of $\Delta$ on $R_{n}$, where $R_{n}$ is, from now on, taken to be a compact Riemannian manifold. As is well known, the set $\left\{\phi_{m}\right\}$ is complete in the $L^{2}$ sense. Furthermore, from the asymptotic behavior of the $\lambda_{m}, \phi_{m}$ (see, for instance, [8]) it follows that for every function $g$ on $R_{n}, g=\sum a_{m} \phi_{m}$ ( $a_{m}$ are Fourier's coefficients) and the series is uniformly convergent together with any preassigned number of its term-by-term derivatives, provided $g$ is sufficiently smooth. We wish to characterize Einsteinian metrics by means of properties of the $\phi_{m}$. Since the $\phi_{m}$, however, are not in general non-negative functions, the above cited theorem is not helpful. We shall instead use the following theorem whose proof, which is similar to that of the above cited theorem, is omitted.

Theorem 6. A necessary and sufficient condition that $R_{n}$ be Einsteinian at $x^{0}$ is that for every solution of $\Delta u+\lambda u=0$ in some $D_{R_{u}},\left(R_{u}<\bar{R}\right.$, for some $\left.\bar{R}>0\right)$

$$
M^{\prime}(R)+\frac{\lambda}{S(R)} \int_{0}^{R} S(r) M(r) d r=\mu(R) O\left(R^{2}\right), \quad \mu(R)=\text { l.u.b. }_{S}|u|
$$

for $R \leqq R_{u}$, where $\left|O\left(R^{2}\right)\right| \leqq A R^{2}$ and $A$ is independent of $u, R_{u}, R$.

REMARK 1. Theorem 6 remains true if $\mu(R)$ is replaced by $\hat{\mu}(R)$ $=$ l.u.b. $D_{R}|u|$. In both cases, the "sufficiency" part remains true if $O\left(R^{2}\right)$ is replaced by $o(R)$. 
REMark 2. From the proof of Theorem 6 it follows that $A$ and $\bar{R}$ are independent of $\lambda$.

THEOREM 7. A necessary and sufficient condition that $R_{n}$ be Einsteinian at $x^{0}$ is that $\left\{\phi_{m}\right\},\left\{\lambda_{m}\right\}$ satisfy for all $R<\bar{R}$ (for some $\bar{R}>0$ )

$$
\frac{\partial}{\partial R} M\left(\phi_{m}, x^{0}, R\right)+\frac{\lambda_{m}}{S(R)} \int_{0}^{R} S(r) M\left(\phi_{m}, x^{0}, r\right) d r=\mu_{m}(R) O\left(R^{2}\right)
$$

where $\mu_{m}(R)=$ l.u.b. $s_{R}\left|\phi_{m}\right|,\left|O\left(R^{2}\right)\right| \leqq A R^{2}$ and $A$ is independent of $m, R$.

Proof. The "necessary" part is a consequence of Theorem 6 and Remark 2. To prove the "sufficiency" part, we need the relation:

$$
S(R) M^{\prime}(R)+M(R) \int_{S_{R}} \Delta R d \sigma=\int_{S_{R}} u \Delta R d \sigma-\lambda \int_{0}^{R} S(r) M(r) d r
$$

valid for any solution of $\Delta u+\lambda u=0$. Its derivation is similar to that of (1.17). Taking $u=\phi_{m}, \lambda=\lambda_{m}$ in (5.4) and making use of (5.3) we obtain

$$
M_{m}(R) \int_{S_{R}} \Delta R d \sigma=\int_{S_{R}} \phi_{m} \Delta R d \sigma+\mu_{m}(R) O\left(R^{2}\right)
$$

where we set $M_{m}(R)=M\left(\phi_{m}, x^{0}, R\right)$. Inserting $\Delta R$ from (1.8) into (5.5) and using the notation (2.29), we get

$$
\begin{aligned}
{\left[\frac{1}{S(R)} \int_{S_{R}} f(\Theta) d \sigma\right]\left[\frac{1}{S(R)} \int_{S_{R}} \phi_{m}(R, \Theta) d \sigma\right] } \\
=\frac{1}{S(R)} \int_{S_{R}} \phi_{m}(R, \Theta) f(\Theta) d \sigma+\mu_{m} O(R)
\end{aligned}
$$

for $0<R<R_{0}$ (for some $R_{0}$ ), where $\mu_{m}=1$.u.b. $0<R<R_{0}\left|\phi_{m}(R)\right|$.

The function $f(\Theta)$ is smooth in some domain containing $\bar{D}_{R_{0}}$, if $R_{0}$ is sufficiently small. Let $\hat{f}$ be a smooth function on $R_{n}$ which coincides with $f$ on $D_{R_{0}}$. Then

$$
\hat{f}=\sum c_{m} \phi_{m},
$$

and the series is uniformly convergent. Hence, for any $\epsilon>0$ there exists a $k$ such that

$$
\left|f-\sum_{m=1}^{k} c_{m} \phi_{m}\right|<\epsilon \text { in } D_{R_{0}} .
$$

We now multiply both sides of (5.6) by $c_{m}$ and sum over $m=1, \cdots, k$. We obtain 


$$
\begin{aligned}
& {\left[\frac{1}{S(R)} \int_{S_{R}} f(\Theta) d \sigma_{R}\right]\left[\frac{1}{S(R)} \int_{S_{R}}\left(f(\Theta)+h_{\epsilon}(R, \Theta)\right) d \sigma_{R}\right]} \\
& \quad=\frac{1}{S(R)} \int_{S_{R}} f(\Theta)\left(f(\Theta)+h_{\epsilon}(R, \Theta)\right) d \sigma_{R}+\left(\sum_{m=1}^{k}\left|c_{m}\right| \mu_{m}\right) O(R)
\end{aligned}
$$

where, for clarity, we set $d \sigma=d \sigma_{R}$ on $S_{R}$, and where $\left|h_{\mathrm{\epsilon}}(R, \Theta)\right|<\epsilon$.

Denoting $d \omega=\lim _{R \rightarrow 0}\left(d \sigma_{R} / S(R)\right)$ and letting $R \rightarrow 0$ in (5.8), we obtain the inequality

$$
\left(\int f(\Theta) d \omega\right)^{2}+\epsilon\left|\int f(\Theta) d \omega\right| \geqq \int(f(\Theta))^{2} d \omega-\epsilon \int|f(\Theta)| d \omega .
$$

Taking $\epsilon \rightarrow 0$, we get

$$
\left(\int f(\Theta) d \omega\right)^{2} \geqq \int(f(\Theta))^{2} d \omega .
$$

Noting that $d \omega>0, \int d \omega=1$, we can use Schwarz's inequality and conclude that $\left(\int f d \omega\right)^{2} \leqq \int f^{2} d \omega$ and equality holds if and only if $f(\Theta) \equiv$ const. In view of (5.9), $f(\Theta)$ is indeed a constant. Hence, by Lemma $1, R_{n}$ is Einsteinian at $x^{0}$.

REMARK. Theorem 7 remains true if, in (5.3), $O\left(R^{2}\right)$ is replaced by $o(R)$.

6. Characterization of harmonic metrics, using solutions of $\Delta u+\lambda u=0$. Using (5.4) one can show that $R_{n}$ is harmonic at $x^{0}$ if and only if

$$
M\left(u, x^{0}, R\right)=\delta(R) u\left(x^{0}\right)
$$

for $R<R_{u}$,

for any solution $u$ of $\Delta u+\lambda u=0$ in $D_{R_{u}}$, where $\delta(R)$ is defined by

$$
\delta^{\prime}(R)+\frac{\lambda}{S(R)} \int_{0}^{R} S(r) \delta(r) d r=0, \quad \delta(0)=1 .
$$

We shall prove:

THEOREM 8. A necessary and sufficient condition that $R_{n}$ be harmonic at $x^{0}$ is that $\left\{\phi_{m}\right\},\left\{\lambda_{m}\right\}$ satisfy, for all $R<\bar{R}$ (for some $\bar{R}>0$ )

$$
M\left(\phi_{m}, x^{0}, R\right)=\delta_{m}(R) \phi_{m}\left(x^{0}\right)
$$

where $\delta_{m}(R)$ is defined by

$$
\delta_{m}^{\prime}(R)+\frac{\lambda_{m}}{S(R)} \int_{0}^{R} S(r) \delta_{m}(r) d r=0, \quad \delta_{m}(0)=1
$$

Proof. We only have to prove the "sufficiency" part of the theorem. By [13] it is enough to prove that

$$
M\left(u, x^{0}, R\right)=u\left(x^{0}\right) \quad \text { for } R<R_{u}
$$


for any solution $u$ of $\Delta u=0$ in $D_{R_{u}}$. Now every local solution can be extended into a smooth function on $R_{n}$. Hence we have

$$
u(x)=\sum_{m=0}^{\infty} a_{m} \phi_{m}(x), \quad \Delta u=-\sum_{m=0}^{\infty} \lambda_{m} a_{m} \phi_{m}(x)=0
$$

uniformly in $x$ for $0 \leqq r \leqq R_{u}^{\prime}$ for any $R_{u}^{\prime}<R_{u}$ ( $r$ is the geodesic distance from $x^{0}$ to $\left.x\right)$.

From (6.6) we obtain

$$
\begin{aligned}
& M\left(u, x^{0}, R\right)=\sum_{m=1}^{\infty} a_{m} M\left(\phi_{m}, x^{0}, R\right)=\sum_{m=1}^{\infty} a_{m} \delta_{m}(R) \phi_{m}\left(x^{0}\right), \\
& M\left(u, x^{0}, R\right)=-\sum_{m=1}^{\infty} \lambda_{m} a_{m} \delta_{m}(R) \phi_{m}\left(x^{0}\right)=0
\end{aligned}
$$

and both series converge uniformly for $0<R<R_{u}^{\prime}$. Multiplying (6.8) by $S(R) / S(\rho)$ and integrating over $R$, we obtain

$$
\sum_{m=1}^{\infty} a_{m} \phi_{m}\left(x^{0}\right)\left[\frac{\lambda_{m}}{S(\rho)} \int_{0}^{\rho} S(R) \delta_{m}(R) d R\right]=0
$$

and the convergence is uniform in $\rho, 0<\rho \leqq R_{u}^{\prime}$.

Using (6.4), (6.9) reduces to

$$
\sum_{m=1}^{\infty} a_{m} \delta_{m}^{\prime}(\rho) \phi_{m}\left(x^{0}\right)=0 .
$$

Finally, integrating the series (6.10), which is uniformly convergent for $0<\rho<R_{u}^{\prime}$, we find that $\sum_{m=1}^{\infty} a_{m} \delta_{m}(R) \phi_{m}\left(x^{0}\right)$ is a function independent of $R$. Hence, recalling (6.7), the proof of (6.5) follows.

REMARK 1. The fundamental solution $\phi(R)$ can be determined, up to an additive constant, by the function $\delta(R)$ defined in (6.2). Indeed, using (2.1) with $\gamma$ defined by (2.6) and $\phi$ being a solution of $\Delta \phi+\lambda \phi=0$, we get

$$
\begin{aligned}
u\left(x^{0}\right)= & -\lambda \gamma_{n} \phi(R) \int_{0}^{R} S(r) M(r) d r-\lambda \gamma_{n} \int_{S_{R}} u \frac{\partial \phi}{\partial \nu} d \sigma \\
& -\int_{D_{R}} \gamma(\Delta+\lambda) u d V .
\end{aligned}
$$

Employing (6.1) and $(\Delta+\lambda) u=0$, we obtain

$$
-\gamma_{n} \frac{d \phi(R)}{d R} \int_{S_{R}} u d \sigma=u\left(x^{0}\right)\left[1+\lambda \gamma_{n} \phi(R) \int_{0}^{R} S(r) \delta(r) d r\right]
$$


Comparing this with (6.1) we get an equation which, after using (6.2), simplifies to

$$
\delta^{\prime}(R) \phi(R)-\delta(R) \phi^{\prime}(R)=\frac{1}{\gamma_{n} S(R)} .
$$

If we can express $S(R)$ in terms of $\phi(R)$, then (6.12) can be used to solve $\phi(R)$ in terms of $\delta(R)$. Now setting $u \equiv 1$ in (6.11) gives

$$
S(R) \phi^{\prime}(R)+\lambda \int_{0}^{R} S(r) \phi(r) d r+\frac{1}{\gamma_{n}}=0
$$

which can be used to express $S(R)$ in terms of $\phi(R)$.

REMARK 2. We have proved in $\S \S 5,6$ that some properties of $\left\{\phi_{m}\right\},\left\{\lambda_{m}\right\}$ determine some properties of the metric of the manifold. A direct construction of the metric from the sequences of eigenvalues and eigenfunctions seems to be hard to accomplish. All we can prove is: If $\left\{\phi_{m}\right\},\left\{\lambda_{m}\right\}$ are the sequences of eigenfunctions and eigenvalues of a metric tensor $\left(g_{i j}\right)$ on a compact Riemannian manifold, then the $\left\{\phi_{m}\right\},\left\{\lambda_{m}\right\}$ determine the metric tensor $\left(g_{i j}\right)$ in a unique manner (i.e., they cannot be the sequences corresponding to another metric tensor).

The proof is based on the following observations:

(a) The $\phi_{m}, \lambda_{m}$ determine the set of all local solutions (that is: in neighborhoods of a point) by the set of all sequences $\left\{a_{m}\right\}$ for which $\sum a_{m} \phi_{m}, \sum \lambda_{m} a_{m} \phi_{m}$ are uniformly (locally) convergent and the second series vanishes.

(b) The local solutions of an elliptic equation determine the coefficients up to a common factor (this follows from [5, proof of Theorem 2]). To find this factor one uses the relations $\Delta \phi_{m}+\lambda_{m} \phi_{m}=0$.

\section{REFERENCES}

1. L. Ásgeirson, Über eine Mittelwertseigenschaft von Lösungen hornogener linearer partieller Differentialgleichungen 2. Ordnung mit konstanten Koeffizienten, Math. Ann. vol. 113 (1937) pp. 321-346.

2. R. Courant and D. Hilbert, Methoden der mathematischen Physik, vol. 2, Berlin, Julius Springer, 1937.

3. G. F. D. Duff, Partial differential equations, University of Toronto Press, 1956.

4. L. P. Eisenhart, Riemannian geometry, Princeton University Press, 1949.

5. A. Friedman, Linear partial differential systems with an additional differential equation at one point, J. Math. Mech. vol. 7 (1958) pp. 173-190.

6. S. Helgason, Differential opcrators on homogeneous spaces, Acta Math. vol. 102 (1960) pp. 239-299.

7. M. A. Lichnerowicz, Equations de Laplace et espaces harmoniques, Premier colloque sur les équations aux dérivées partielles, Louvain (1953) pp. 9-23. Paris, Masson, 1954. 
8. S. Minakshisundaram, Eigenfunctions on Riemannian manifolds, J. Indian Math. Soc. vol. 17 (1953) pp. 159-165.

9. J. Nash, Continuity of solutions of parabolic and elliptic equations, Amer. J. Math. vol. 80 (1958) pp. 931-954.

10. T. Y. Thomas and E. W. Titt, On the elementary solution of the general linear differential equation of the second order with analytic coefficients, J. Math. Pures Appl. vol. 18 (1939) pp. 217-248.

11. O. Veblen, Invariants of quadratic differential forms, Cambridge University Press, 1927.

12. 'T. J. Willmore, Some properties of harmonic Riemannian manifolds, Trieste, Convegno Internaz. Geometria Differenziale Italia, 1953, pp. 141-147.

13. - - Mean value theorems, J. London Math. Soc. vol. 25 (1950) pp. 54-57.

14. - An introduction to differential geometry, Oxford University Press, 1959.

University of Minnesota, Institute of Technology, MinNeAPOLIS, MinNESOTA 